\title{
The molecular clump towards the eastern border of SNR G18.8+0.3
}

\author{
S. Paron ${ }^{1,2,3}$, M. E. Ortega ${ }^{1}$, A. Petriella ${ }^{1,3}$, M. Rubio ${ }^{4}$, G. Dubner $^{1}$, and E. Giacani ${ }^{1,2}$ \\ ${ }^{1}$ Instituto de Astronomía y Física del Espacio (IAFE), CC 67, Suc. 28, 1428 Buenos Aires, Argentina \\ e-mail: sparon@iafe.uba.ar \\ 2 FADU - Universidad de Buenos Aires, Ciudad Universitaria, Buenos Aires, Argentina \\ 3 CBC - Universidad de Buenos Aires, Ciudad Universitaria, Buenos Aires, Argentina \\ ${ }^{4}$ Departamento de Astronomía, Universidad de Chile, Casilla 36-D, Santiago, Chile
}

Received 5 July 2012 / Accepted 7 September 2012

\section{ABSTRACT}

\begin{abstract}
Aims. The eastern border of the SNR G18.8+0.3, close to an HII regions complex, is a very interesting region for studying the molecular gas that it is probably in contact with the supernova remnant (SNR) shock front.

Methods. We observed this region using the Atacama Submillimeter Telescope Experiment (ASTE) in the ${ }^{12} \mathrm{CO} J=3-2,{ }^{13} \mathrm{CO}$ $J=3-2, \mathrm{HCO}^{+} J=4-3$, and CS $J=7-6$ lines with an angular resolution of $22^{\prime \prime}$. To complement these observations, we analyzed infrared, submillimeter, and radio continuum archival data.

Results. In this work, we clearly show that the radio continuum "protrusion" that was earlier thought to belong to the SNR is an HII region complex that is deeply embedded in a molecular clump. The new molecular observations reveal that this dense clump, belonging to an extended molecular cloud that surrounds the SNR's southeast border, is not physically in contact with SNR G18.8+0.3, suggesting that the SNR shock front has not yet reached it or that they may be located at different distances. We found some young stellar objects embedded in the molecular clump, suggesting that their formation should be approximately coeval with the SN explosion.
\end{abstract}

Key words. ISM: clouds - ISM: supernova remnants - stars: formation

\section{Introduction}

The conversion of gas into stars involves a diversity of objects (molecular clouds, dust, magnetic fields, etc.) and several highly nonlinear and multidimensional dynamical processes (turbulence, self-gravity, etc.) operating on different scales, which are still far from understood in spite of all the theoretical and observational advances. Detailed multiwavelength studies on different spatial scales from large clouds to star embryos are very helpful for understanding precisely how the gas and dust coalesce until forming new stars. In particular, investigating the properties of the medium from which stars form is a useful way to know the initial conditions that favor star formation and the most favorable mechanisms for triggering the process.

In this context, the molecular cloud is supposedly interacting with the SNR G18.8+0.3 (Dubner et al. 1999, 2004; Tian et al. 2007), which harbors IRAS pointlike sources compatible with the characteristics of protostellar candidates (IRAS sources whose colors correspond to those of ultracompact HII regions, i.e. sites of recent massive star formation Wood \& Churchwell 1989), is an interesting target. Studying it allows us not only to analyze the properties of the interstellar matter around pre stars, but also to explore whether there is some relation between the newborn stars and the SNR. Studies of the molecular gas in regions suspected of being stellar nurseries, performed with intermediate spatial resolution, are especially useful for pinpointing the best candidates for pursuing the millimetric and submillimetric studies with the unprecedented resources provided by ALMA.

The SNR G18.8+0.3 has a peculiar morphology with the eastern and southern flanks strongly flattened, while it fades to the west (Fig. 1), suggesting a marked density gradient in

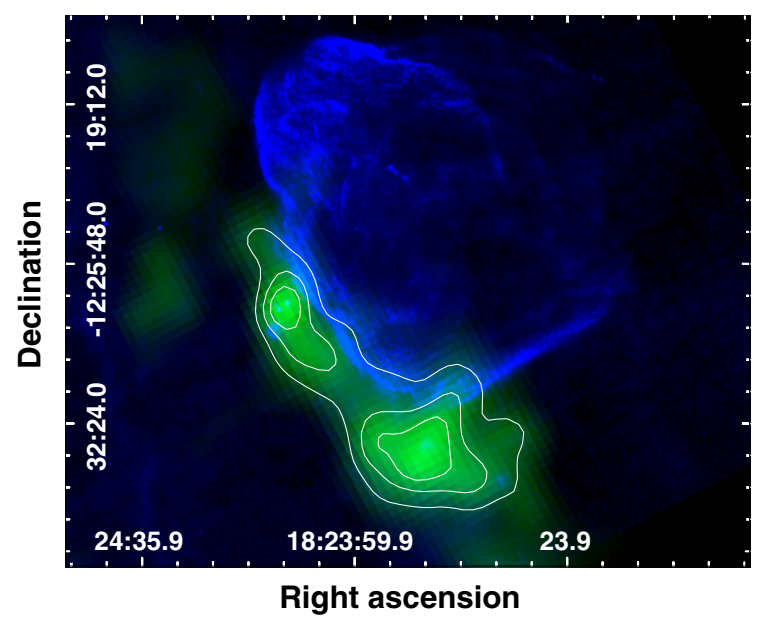

Fig. 1. The SNR G18.8+0.3 radio continuum emission at $20 \mathrm{~cm}$ is displayed in blue and the ${ }^{13} \mathrm{CO} J=1-0$ emission, extracted from the GRS (Simon et al. 2001), which was averaged between 17 and $22 \mathrm{~km} \mathrm{~s}^{-1}$ is presented in green with withe contours.

the ambient gas. Based on VLA radio continuum observations, Dubner et al. (1996) pointed out the existence of a "protrusion" emerging from the eastern border of the SNR, near $18^{\mathrm{h}} 24^{\mathrm{m}} 14^{\mathrm{s}}$, $-12^{\circ} 28^{\prime} 30^{\prime \prime}(\mathrm{J} 2000)$. Subsequent observations performed with better angular resolution resolved, as described below, this region into several HII regions. In this paper, we analyze the molecular emission in this spot.

Arnal et al. (1993) reported the detection of ${ }^{12} \mathrm{CO}$ line emission along the whole eastern border of the SNR G18.8+0.3 suggesting that the cloud might have been shocked by the expanding 


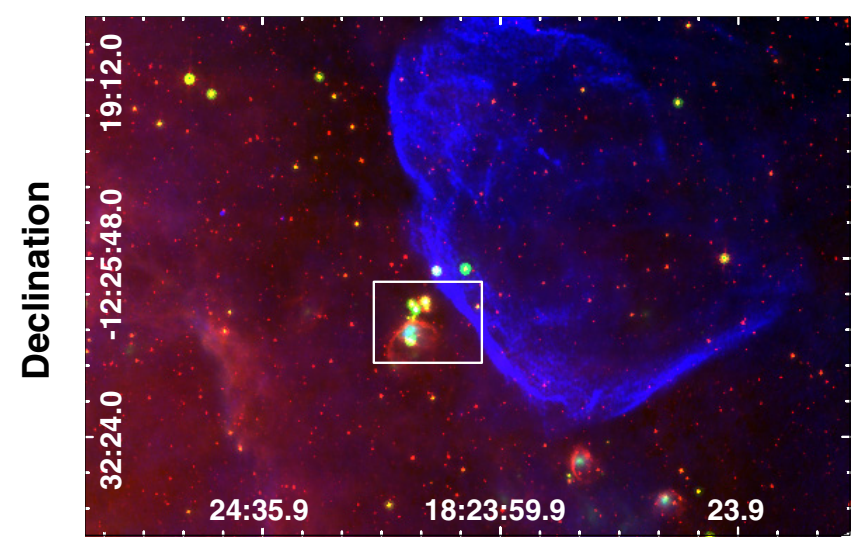

Right ascension

Fig. 2. Three-color image of the SNR G18.8+0.3 and its surroundings. Again the radio continuum emission at $20 \mathrm{~cm}$ is presented in blue, the infrared bands at $8 \mu \mathrm{m}$ and $24 \mu \mathrm{m}$ are shown in red and green, respectively. The white rectangle shows the studied region.

remnant. Later, Dubner et al. (1999) carried out observations in $\mathrm{HI},{ }^{12} \mathrm{CO}$, and ${ }^{13} \mathrm{CO}$ in the direction to $\mathrm{G} 18.8+0.3$, concluding that the SNR explosion took place near the border of a preexisting cloud, driving a slow shock $\left(v \sim 10 \mathrm{~km} \mathrm{~s}^{-1}\right)$ into the cloud. Based on the systemic velocity $v_{\mathrm{LSR}} \sim 19 \mathrm{~km} \mathrm{~s}^{-1}$ of the interacting molecular cloud, the distances of 1.9 and $14.1 \mathrm{kpc}$ were estimated by applying a Galactic circular rotation model. In that paper, the distance ambiguity was wrongly interpreted by favoring the closest option, a problem solved in Dubner et al. (2004), where the far distance of $14 \mathrm{kpc}$ was confirmed for G18.8+0.3. More recently, based on ${ }^{13} \mathrm{CO}$ emission and $\mathrm{HI}$ absorption techniques, Tian et al. (2007) have established $6.9 \mathrm{kpc}$ and $15 \mathrm{kpc}$ as the lower and upper limits for the distance to this SNR.

\section{Presentation of the investigated region}

Figure 1 shows the SNR G18.8+0.3 as seen in the radio continuum emission at $20 \mathrm{~cm}$ extracted from the MAGPIS (Helfand et al. 2006) and its molecular environment in the ${ }^{13} \mathrm{CO} J=1-0$ emission averaged between 17 and $22 \mathrm{~km} \mathrm{~s}^{-1}$. The ${ }^{13} \mathrm{CO}$ data, with an angular resolution of $466^{\prime \prime}$, were extracted from the Galactic Ring Survey (GRS; Simon et al. 2001). The image clearly shows a large-scale morphological correspondence between the SNR southeastern border and the molecular gas, strongly suggesting an interaction between them, as has been suggested in previous works. Figure 2 shows the SNR G18.8+0.3 radio continuum and its infrared (IR) environment as seen in the Spitzer-IRAC band at $8 \mu \mathrm{m}$, and the Spitzer-MIPS band at $24 \mu \mathrm{m}$. Figure 3 displays a zoom-in of this region showing the presence of several cataloged radio sources. The source $\mathrm{RS} 1$ is cataloged in the HII Region Discovery Survey (Anderson et al. 2011) as an irregular bubble $(\mathrm{G} 18.751+0.254)$ with a recombination line at the LSR velocity $19.1 \mathrm{~km} \mathrm{~s}^{-1}$, thus confirming that it is immersed in the same molecular cloud adjacent to the SNR G18.8+0.3. In Fig. 3, it can be appreciated the radio continuum emission of this HII region surrounded by $8 \mu \mathrm{m}$ IR emission with a bright center emitting in $24 \mu \mathrm{m}$, as is usually observed in infrared dust bubbles (Churchwell et al. 2006, 2007). The sources RS2, RS3, and RS4 appear as discrete radio sources in the MAGPIS $20 \mathrm{~cm}$ Survey. In particular the source RS4, cataloged as a radio-compact HII region (Giveon et al. 2005), lies at the same position as the $870 \mu \mathrm{m}$ continuum source G18.76+0.26.

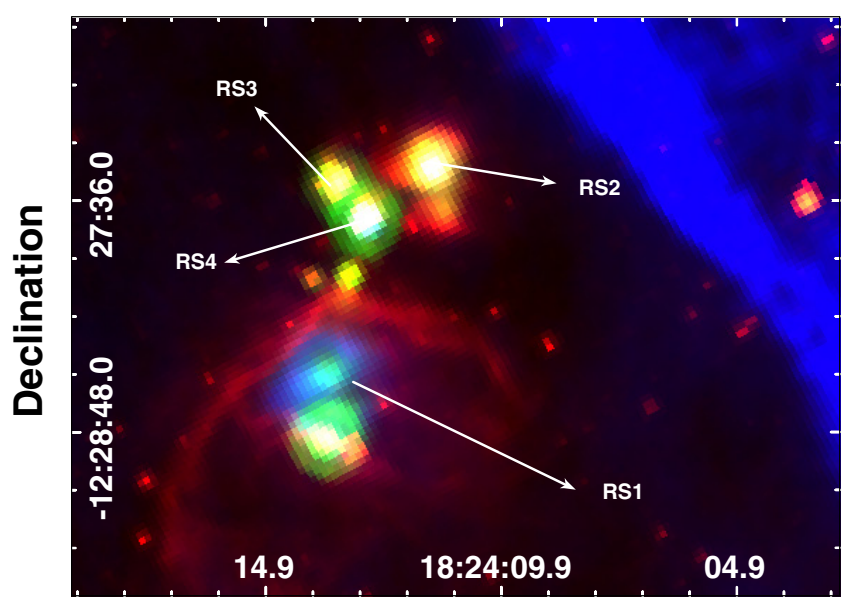

Right ascension

Fig. 3. Three-color image of the region marked with the white box in Fig. 2. Radio continuum at $20 \mathrm{~cm}=$ blue, $8 \mu \mathrm{m}=$ red, and $24 \mu \mathrm{m}=$ green. The cataloged radio sources described in the text are marked.

This source, observed with the Large APEX Bolometer Camera (LABOCA) and cataloged in the ATLASGAL, has an angular extension of $59^{\prime \prime} \times 42^{\prime \prime}$ (Schuller et al. 2009). From observations of the $\mathrm{NH}_{3}(1,1)$ line, these authors report a $v_{\mathrm{LSR}}=20.8 \mathrm{~km} \mathrm{~s}^{-1}$ for $\mathrm{G} 18.76+0.26$ and conclude that this source is located at the distance of about $14 \mathrm{kpc}$.

We conclude that the molecular complex abutting the eastern border of the SNR G18.8+0.3 is a very rich region populated by several HII regions. The HI absorption analysis performed by Tian et al. (2007) towards this region (region 6 in their work) gives a spectrum with the same absorption features as the spectra towards several regions over the SNR, strongly suggesting that this HII region complex is located at the same distance as the SNR. Thus, we adopt a distance of about $14 \pm 1 \mathrm{kpc}$ for the SNR, the molecular cloud, and the HII region complex. The error bar of $2 \mathrm{kpc}$ comes by considering noncircular motions in the Galactic rotation model towards this region of the Galaxy.

\section{Observations}

The molecular observations presented in this work were performed on June 12 and 13, 2011 with the $10 \mathrm{~m}$ Atacama Submillimeter Telescope Experiment (ASTE; Ezawa et al. 2004). We used the CATS $345 \mathrm{GHz}$ band receiver, which is a two-single band SIS receiver remotely tunable in the LO frequency range of $324-372 \mathrm{GHz}$. We simultaneously observed ${ }^{12} \mathrm{CO} J=3-2$ at $345.796 \mathrm{GHz}$ and $\mathrm{HCO}^{+} J=4-3$ at $356.734 \mathrm{GHz}$, mapping a region of $240^{\prime \prime} \times 150^{\prime \prime}$ centered at $\mathrm{RA}=18^{\mathrm{h}} 24^{\mathrm{m}} 10.9^{\mathrm{s}}$, Dec $=-12^{\circ} 28^{\prime} 22.0^{\prime \prime}, \mathrm{J} 2000$. We also observed ${ }^{13} \mathrm{CO} J=3-2$ at $330.588 \mathrm{GHz}$ and $\mathrm{CS} J=7-6$ at $342.883 \mathrm{GHz}$ mapping a region of $120^{\prime \prime} \times 120^{\prime \prime}$ centered at RA $=18^{\mathrm{h}} 24^{\mathrm{m}} 10.9^{\mathrm{s}}$, Dec $=-12^{\circ} 27^{\prime} 20.0^{\prime \prime}$. The mapping grid spacing was $20^{\prime \prime}$ in all cases and the integration time was 30 and $60 \mathrm{~s}$ per pointing in each case, respectively. All the observations were performed in position switching mode.

We used the XF digital spectrometer with a bandwidth set to $128 \mathrm{MHz}$ and spectral resolution set to $125 \mathrm{kHz}$. The velocity resolution was $0.11 \mathrm{~km} \mathrm{~s}^{-1}$ and the half-power beamwidth (HPBW) was 22" at $345 \mathrm{GHz}$. The weather conditions were optimal, and the system temperature varied from $T_{\text {sys }}=150$ to $200 \mathrm{~K}$. The main beam efficiency was $\eta_{\mathrm{mb}} \sim 0.65$. All quoted numbers for the line temperatures along this work were 


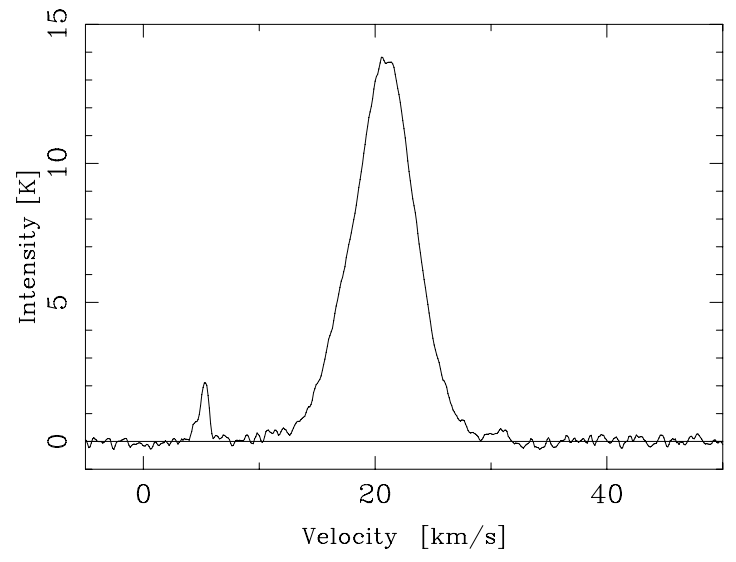

Fig. 4. ${ }^{12} \mathrm{CO} J=3-2$ spectrum obtained towards the offset $\left(+20^{\prime \prime},+20^{\prime \prime}\right)$ position relative to $\mathrm{RA}=18^{\mathrm{h}} 24^{\mathrm{m}} 10.9^{\mathrm{s}}$, Dec $=-12^{\circ} 28^{\prime} 22.0^{\prime \prime}, \mathrm{J} 2000$.

corrected for the antenna efficiency; i.e., in all cases, they are the main brightness temperature. The spectra were Hanning smoothed to improve the signal-to-noise ratio $(\mathrm{S} / \mathrm{N})$ and only linear or/and some third order polynomia were used for baseline fitting. The data were reduced with NEWSTAR ${ }^{1}$ and the spectra processed using the XSpec software package ${ }^{2}$.

\section{Molecular gas}

Figure 4 displays a typical ${ }^{12} \mathrm{CO} J=3-2$ spectrum obtained towards the surveyed region. This spectrum, corresponding to the offset position $\left(+20^{\prime \prime},+20^{\prime \prime}\right)$, exhibits two components: a weak one at $\sim 5 \mathrm{~km} \mathrm{~s}^{-1}$, and the main component centered at $\sim 19 \mathrm{~km} \mathrm{~s}^{-1}$. The weak component corresponds to local gas emission and will not be further considered. The main component goes from $\sim 10$ to $30 \mathrm{~km} \mathrm{~s}^{-1}$, and represents the molecular gas that it is very likely related to the SNR and the HII regions complex. Figure 5 shows the gas distribution as seen in the ${ }^{12} \mathrm{CO} J=3-2$ emission integrated every $2 \mathrm{~km} \mathrm{~s}^{-1}$ from 14 to $32 \mathrm{~km} \mathrm{~s}^{-1}$. After integrating the ${ }^{12} \mathrm{CO} J=3-2$ emission from 10 to $30 \mathrm{~km} \mathrm{~s}^{-1}$, we obtain the molecular clump shown in Fig. 6 . The HII region G18.751+0.254 (source RS1 in Fig. 3 seen in blue in Fig. 4) appears surrounded by the molecular emission, whose peak coincides with the region where the sources RS2, RS3, and RS4 are located. Considering that the southeastern border of SNR G18.8+0.3 is surrounded by an extended molecular cloud detected in lower molecular transitions (see Fig. 1), we conclude that with the present observations we are analyzing a molecular clump belonging to this extended cloud.

From the inspection of the other observed molecular transitions, we find that the $\mathrm{HCO}^{+} J=4-3$ and ${ }^{13} \mathrm{CO} J=3-2$ lines are only bright near the peak of the molecular clump, i.e. towards the densest region. Figures 7 and 8 display the $\mathrm{HCO}^{+} J=4-3$ and ${ }^{13} \mathrm{CO} J=3-2$ emissions, respectively, integrated between 10 and $30 \mathrm{~km} \mathrm{~s}^{-1}$.

Based on the presented molecular maps we suggest that RS1 has shaped the surrounding gas, while the other radio sources, likely younger HII regions, are still embedded in the densest portion of the molecular clump. On the other hand, it is important to note that the analyzed molecular clump is not in physical contact

\footnotetext{
Reduction software based on AIPS developed at NRAO, extended to treat single-dish data with a graphical user interface (GUI).

2 XSpec is a spectral line reduction package for astronomy that has been developed by Per Bergman at Onsala Space Observatory.
}
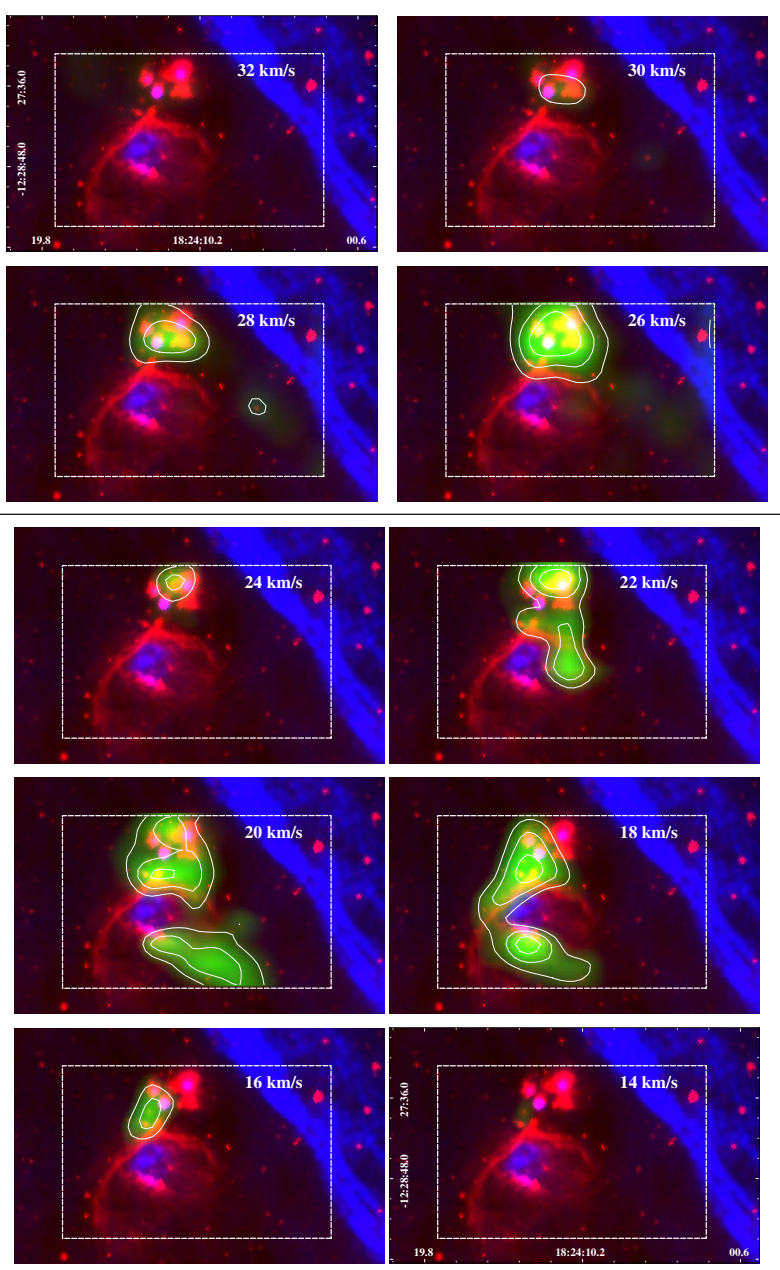

Fig. 5. Integrated velocity channel maps of the ${ }^{12} \mathrm{CO} J=3-2$ emission every $2 \mathrm{~km} \mathrm{~s}^{-1}$ (in green). As in previous figures, is shown the IRAC emission at $8 \mu \mathrm{m}$ in red and the radio continuum emission at $20 \mathrm{~cm}$ in blue. The contours levels in the upper panels (from 32 to $26 \mathrm{~km} \mathrm{~s}^{-1}$ ) are $1.8,3.6$, and $7.0 \mathrm{~K} \mathrm{~km} \mathrm{~s}^{-1}$, while the contours levels in the bottom panels (from 24 to $14 \mathrm{~km} \mathrm{~s}^{-1}$ ) are $11.5,15.0$, and $19.5 \mathrm{~K} \mathrm{~km} \mathrm{~s}^{-1}$. The dashed rectangle shows the mapped region.

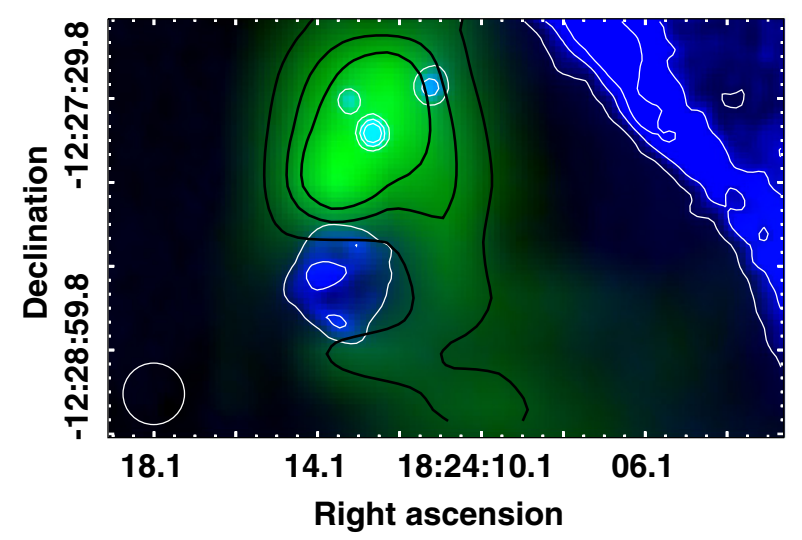

Fig. 6. The ${ }^{12} \mathrm{CO} J=3-2$ emission integrated between 10 and $30 \mathrm{~km} \mathrm{~s}^{-1}$ is presented in green with black contours with levels of 46,65 , and $80 \mathrm{~K} \mathrm{~km} \mathrm{~s}^{-1}$. The radio continuum emission at $20 \mathrm{~cm}$ is shown in blue with white contours of $2.3,6$, and $11 \mathrm{mJy} \mathrm{beam}^{-1}$. The angular resolutions are about $20^{\prime \prime}$ and 6 " for the molecular and radio continuum emissions, respectively. The beam of the molecular observations is included towards the bottom left corner. 


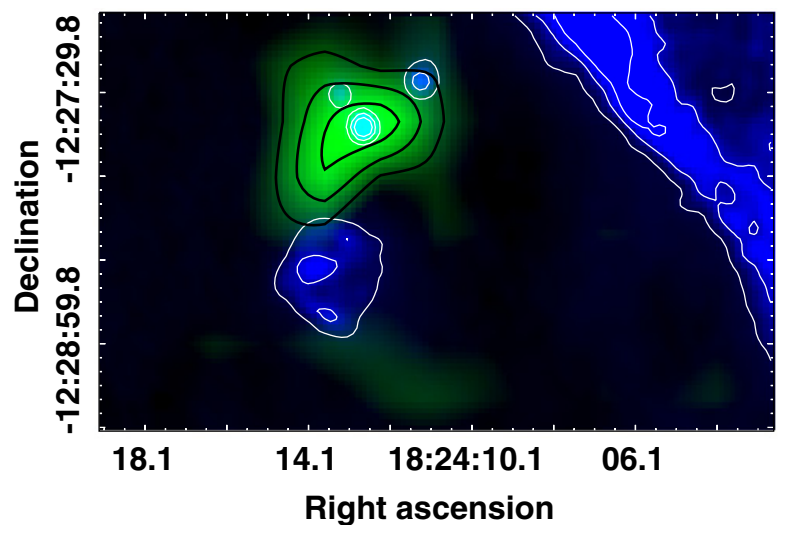

Fig. 7. The $\mathrm{HCO}^{+} J=4-3$ emission integrated between 10 and $30 \mathrm{~km} \mathrm{~s}^{-1}$ is displayed in green with black contours with levels of 4 , 6 , and $8 \mathrm{~K} \mathrm{~km} \mathrm{~s}^{-1}$. The radio continuum emission at $20 \mathrm{~cm}$ is shown in blue with white contours of $2.3,6$, and $11 \mathrm{mJy}^{\text {beam }}{ }^{-1}$.

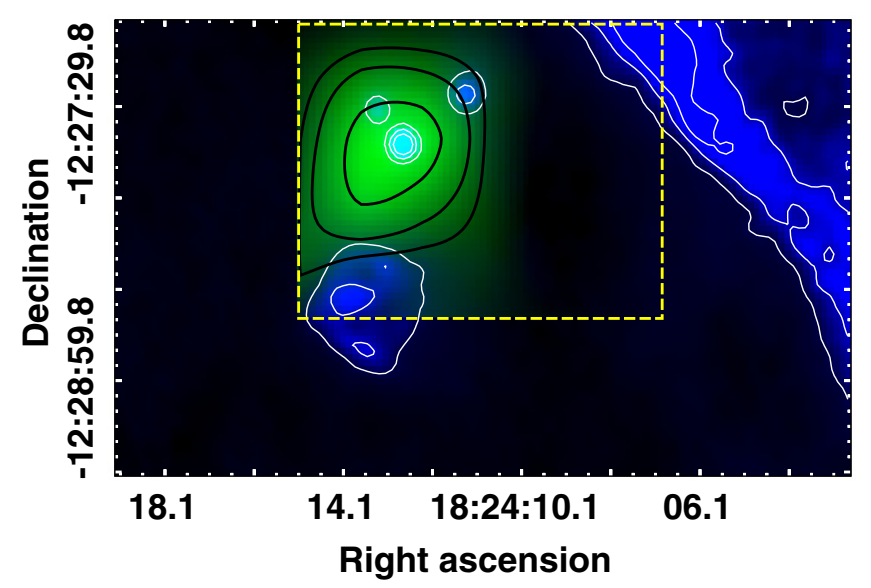

Fig. 8. The ${ }^{13} \mathrm{CO} J=3-2$ emission integrated between 10 and $30 \mathrm{~km} \mathrm{~s}^{-1}$ is presented in green with black contours with levels of 15, 20, and $30 \mathrm{~K} \mathrm{~km} \mathrm{~s}^{-1}$. The radio continuum emission at $20 \mathrm{~cm}$ is shown in blue with white contours of $2.3,6$, and $11 \mathrm{mJy}^{\text {beam }}{ }^{-1}$. The dashed yellow rectangle represents the region surveyed in this line.

with the SNR G18.8+0.3 border, suggesting that the SNR shock front have not yet reached it, or the clump is located at a different distance.

Figure 9 shows the spectra of each molecular species obtained towards the peak position of the molecular clump. The parameters determined from Gaussian fitting of these lines are presented in Table 1, where $T_{\mathrm{mb}}$ represents the peak brightness temperature, $V_{\mathrm{LSR}}$ the central velocity referred to the local standard of rest, and $\Delta v$ the FWHM line width. Errors are formal $1 \sigma$ value for the model of the Gaussian line shape. Additionally, the integrated intensities (I) are included in this table. It is noticeable that the $\mathrm{HCO}^{+} J=4-3$ line appears significantly narrower than the $\mathrm{CO}$ lines, suggesting that the dense gas, mapped by the $\mathrm{HCO}^{+}$, occupies a different volume than the gas mapped by the ${ }^{12} \mathrm{CO}$ and ${ }^{13} \mathrm{CO}$ lines. Regarding the $\mathrm{CS} J=7-6$ line, the data present some hints of emission towards this region. However, the poor $\mathrm{S} / \mathrm{N}$ achieved in the observations of this transition does not allow us to perform a trustable analysis about this molecular species. The CS spectrum presented in Fig. 9, which was obtained towards the peak of the molecular clump, has a $\mathrm{S} / \mathrm{N}$ of about 2.2.

\subsection{Column densities and abundances}

To estimate the molecular column densities and abundances towards the clump, we assume local thermodynamic equilibrium (LTE) and a beam filling factor of 1 . From the peak temperature ratio between the CO isotopes $\left({ }^{12} T_{\mathrm{mb}} /{ }^{13} T_{\mathrm{mb}}\right)$, it is possible to estimate the optical depths from (e.g. Scoville et al. 1986)

$\frac{{ }^{12} T_{\mathrm{mb}}}{{ }^{13} T_{\mathrm{mb}}}=\frac{1-\exp \left(-\tau_{12}\right)}{1-\exp \left(-\tau_{12} / X\right)}$

where $\tau_{12}$ is the optical depth of the ${ }^{12} \mathrm{CO}$ gas and $X=\left[{ }^{12} \mathrm{CO}\right] /\left[{ }^{13} \mathrm{CO}\right]$ is the isotope abundance ratio. The $\left[{ }^{12} \mathrm{CO}\right] /\left[{ }^{13} \mathrm{CO}\right]$ ratio can be estimated from the relation $\left[{ }^{12} \mathrm{C}\right] /\left[{ }^{13} \mathrm{C}\right]=6.21 \times D_{\text {G.C. }}+18.77$ (Milam et al. 2005), where $D_{\mathrm{G} . \mathrm{C}}=6.9 \mathrm{kpc}$ is the distance between the source and the Galactic center, yielding $\left[{ }^{12} \mathrm{C}\right] /\left[{ }^{13} \mathrm{C}\right]=61 \pm 14$. According to Milam et al. (2005) and Savage et al. (2002), the $\left[{ }^{12} \mathrm{C}\right] /\left[{ }^{13} \mathrm{C}\right]$ isotope ratio exhibits a noticeable gradient with distance from the Galactic center, i.e. is strongly dependent with $D_{\mathrm{G} . C .}$. Then, from Eq. (1), the ${ }^{12} \mathrm{CO} J=3-2$ optical depth is $\tau_{12} \sim 30$, while the ${ }^{13} \mathrm{CO} J=3-2$ optical depth is $\tau_{13} \sim 0.5$, revealing that the ${ }^{12} \mathrm{CO}$ line appears optically thick, while the ${ }^{13} \mathrm{CO}$ line is optically thin. Thus, we calculate the excitation temperature from

$$
T_{\text {ex }}(3 \rightarrow 2)=\frac{16.95 \mathrm{~K}}{\ln \left[1+16.59 \mathrm{~K} /\left(T_{\max }\left({ }^{12} \mathrm{CO}\right)+0.036 \mathrm{~K}\right)\right]}
$$

obtaining $T_{\mathrm{ex}} \sim 20 \mathrm{~K}$. Then, we derive the ${ }^{12} \mathrm{CO}$ column density from

$N\left({ }^{12} \mathrm{CO}\right)=7.96 \times 10^{13} \mathrm{e}^{\frac{16.6}{T_{\text {ex }}}} \frac{T_{\text {ex }}+0.92}{1-\exp \left(\frac{-16.6}{T_{\text {ex }}}\right)} \int \tau_{12} \mathrm{dv}$

where, taking into account that $\tau \geq 1$, we use the approximation

$$
\int \tau \mathrm{dv}=\frac{1}{J\left(T_{\mathrm{ex}}\right)-J\left(T_{\mathrm{BG}}\right)} \frac{\tau}{1-\mathrm{e}^{-\tau}} \int T_{\mathrm{mb}} \mathrm{d} v
$$

with

$$
J(T)=\frac{h v / k}{\exp \left(\frac{h v}{k T}\right)-1} .
$$

Finally we obtain $N\left({ }^{12} \mathrm{CO}\right) \sim 1.5 \times 10^{18} \mathrm{~cm}^{-2}$. In the case of the ${ }^{13} \mathrm{CO} J=3-2$ line, we use

$$
N\left({ }^{13} \mathrm{CO}\right)=8.28 \times 10^{13} \mathrm{e}^{\frac{15.87}{T_{\mathrm{ex}}}} \frac{T_{\mathrm{ex}}+0.88}{1-\exp \left(\frac{-15.87}{T_{\mathrm{ex}}}\right)} \int \tau_{13} \mathrm{dv}
$$

and taking into account that this line appears optically thin, we use the approximation

$$
\int \tau \mathrm{dv}=\frac{1}{J\left(T_{\mathrm{ex}}\right)-J\left(T_{\mathrm{BG}}\right)} \int T_{\mathrm{mb}} \mathrm{d} v
$$

$$
\text { yielding } N\left({ }^{13} \mathrm{CO}\right) \sim 1.8 \times 10^{16} \mathrm{~cm}^{-2} \text {. }
$$

The $\mathrm{HCO}^{+}$column density was derived from

$$
N\left(\mathrm{HCO}^{+}\right)=5.85 \times 10^{10} \mathrm{e}^{\frac{25.7}{T_{\mathrm{ex}}}} \frac{T_{\mathrm{ex}}+0.71}{1-\exp \left(\frac{-17.12}{T_{\text {ex }}}\right)} \int \tau \mathrm{dv},
$$

and by assuming that the $\mathrm{HCO}^{+} J=4-3$ is optically thin, we use the same approximation as used for the ${ }^{13} \mathrm{CO}$ line (Eq. (7)). As excitation temperatures we use the range $20-50 \mathrm{~K}$, obtaining $N\left(\mathrm{HCO}^{+}\right) \sim(3-5) \times 10^{12} \mathrm{~cm}^{-2}$. 

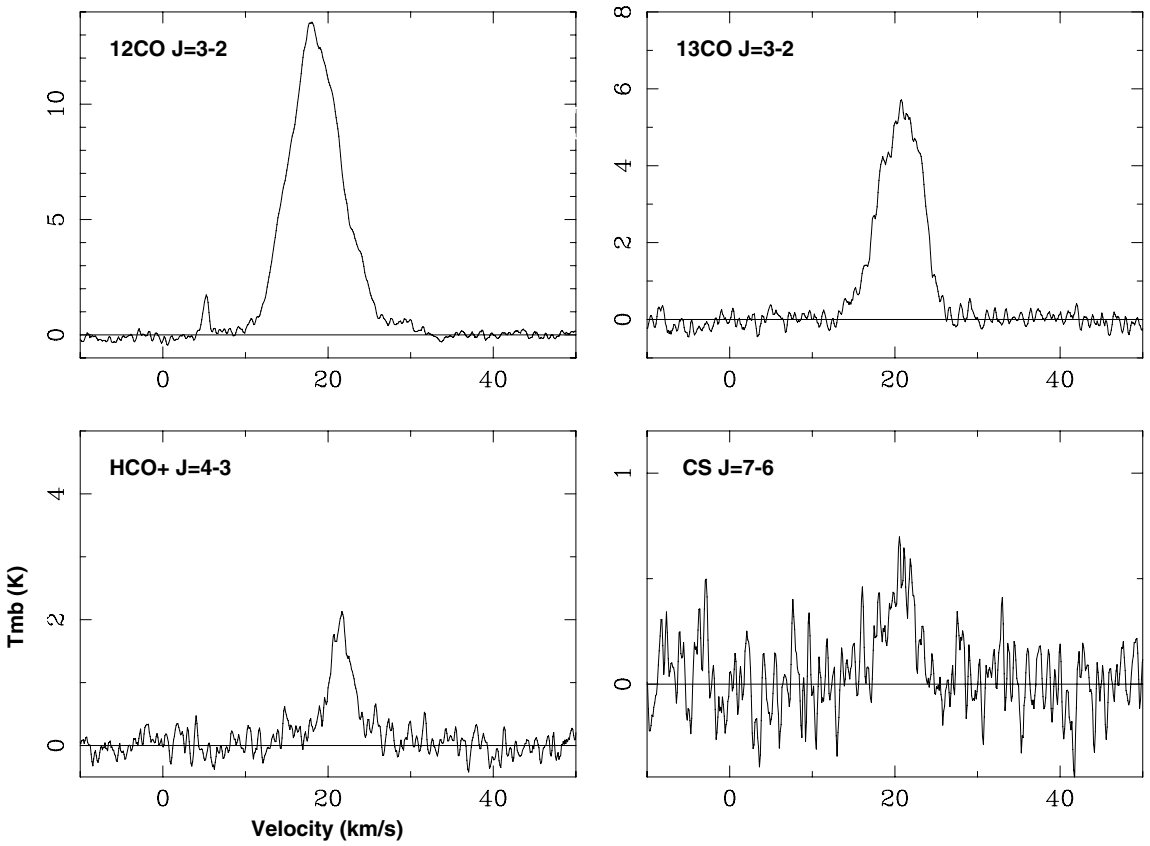

Fig. 9. Spectra of each molecular species obtained towards the molecular clump peak. The ${ }^{12} \mathrm{CO},{ }^{13} \mathrm{CO}$, and $\mathrm{HCO}^{+}$lines have very high $\mathrm{S} / \mathrm{N}$, while the CS emission has an $\mathrm{S} / \mathrm{N}$ of about 2.2.

Table 1. Observed and derived parameters of the molecular lines shown in Fig. 9.

\begin{tabular}{lcccc}
\hline \hline Emission & $\begin{array}{c}T_{\mathrm{mb}} \\
(\mathrm{K})\end{array}$ & $\begin{array}{c}V_{\mathrm{LSR}} \\
\left(\mathrm{km} \mathrm{s}^{-1}\right)\end{array}$ & $\begin{array}{c}\Delta v \\
\left(\mathrm{~km} \mathrm{~s}^{-1}\right)\end{array}$ & $\begin{array}{c}I \\
\left(\mathrm{~K} \mathrm{~km} \mathrm{~s}^{-1}\right)\end{array}$ \\
\hline${ }^{12} \mathrm{CO} J=3-2$ & $12.9 \pm 0.1$ & $18.5 \pm 0.5$ & $7.4 \pm 0.1$ & $104.7 \pm 1.7$ \\
${ }^{13} \mathrm{CO} J=3-2$ & $5.5 \pm 0.2$ & $20.7 \pm 0.5$ & $6.0 \pm 0.2$ & $35.0 \pm 1.8$ \\
$\mathrm{HCO}^{+} J=4-3$ & $1.8 \pm 0.3$ & $21.5 \pm 0.7$ & $3.6 \pm 0.5$ & $9.0 \pm 1.6$ \\
\hline
\end{tabular}

To estimate the molecular abundances it is necessary to have an $\mathrm{H}_{2}$ column density value. As shown in detail in the next section (Sect. 5), we independently estimate this parameter through the millimeter continuum emission, obtaining $N\left(\mathrm{H}_{2}\right) \sim 4.4 \times$ $10^{22} \mathrm{~cm}^{-2}$. By using this value, we obtain the following molecular abundances: $X\left({ }^{12} \mathrm{CO}\right) \sim 3.4 \times 10^{-5}, X\left({ }^{13} \mathrm{CO}\right) \sim 4.1 \times 10^{-7}$, and $X\left(\mathrm{HCO}^{+}\right) \sim(0.7-1) \times 10^{-10}$. The obtained $\mathrm{HCO}^{+}$abundance is very similar to the values derived by Cortes et al. (2010) and Cortes (2011) towards high-mass star-forming regions.

\section{Millimeter continuum emission}

We have also investigated the millimeter dust continuum emission at $1.1 \mathrm{~mm}$ using data from the Bolocam Galactic Plane Survey. We found a source in positional coincidence with the ${ }^{12} \mathrm{CO}$ emission peak, BGPS 18.763+00.261 (Rosolowsky et al. $2010)$, which has a roughly elliptical morphology $\left(41^{\prime \prime} \times 29^{\prime \prime}\right)$. In Fig. 10, we present a two-color image of the BGPS $1.1 \mathrm{~mm}$ emission and the radio continuum emission at $20 \mathrm{~cm}$. The positional agreement between the BGPS source and the molecular emission described above shows that the $1.1 \mathrm{~mm}$ emission originates in the densest portion of the molecular clump.

We estimate the mass of the molecular gas associated with the BGPS source using the equations from Bally et al. (2010), $M\left(\mathrm{H}_{2}\right)=14.26 D^{2} S_{1.1}\left(\mathrm{e}^{13 / T_{\mathrm{d}}}-1\right) M_{\odot}$, where $D$ is the distance in kpc, $S_{1.1}$ is the flux density at $1.1 \mathrm{~mm}$ in $\mathrm{Jy}$, and $T_{\mathrm{d}}$ is the

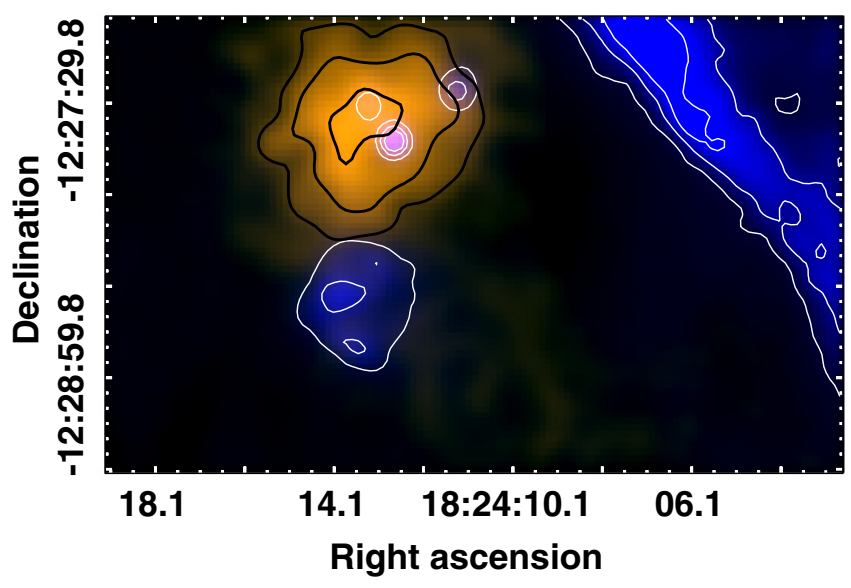

Fig. 10. The smoothed $1.1 \mathrm{~mm}$ dust continuum emission is presented in orange with black contours. The radio continuum emission at $20 \mathrm{~cm}$ is shown in blue with white contours of $2.3,6$, and $11 \mathrm{mJy} \mathrm{beam}^{-1}$.

dust temperature in $\mathrm{K}$. Assuming that the dust and the gas are collisionally coupled, we can approximate $T_{\mathrm{d}}=T_{\mathrm{K}}$, where $T_{\mathrm{K}}$ is the temperature of the gas. For $S_{1.1}$, we use the $80^{\prime \prime}$ aperture flux density, which seems appropriate for the dimensions of this particular BGPS source. The flux value reported in Rosolowsky et al. (2010) is scaled with a calibration factor of 1.5 (Dunham et al. 2011). Thus, by adopting $D=14 \pm 1 \mathrm{kpc}, T_{\mathrm{d}}=20 \mathrm{~K}$, and $S_{1.1}=2.21 \mathrm{Jy}$ we obtain $M\left(\mathrm{H}_{2}\right)=5700 \pm 800 M_{\odot}$. In addition, we estimate the column density of the molecular gas by applying $N\left(\mathrm{H}_{2}\right)=2 \times 10^{22} S_{1.1} \mathrm{~cm}^{-2}$, to obtain $N\left(\mathrm{H}_{2}\right) \sim 4.4 \times 10^{22} \mathrm{~cm}^{-2}$.

\section{The compact radio sources embedded in the molecular gas}

Figure 11 shows a three-color image (blue = radio continuum at $20 \mathrm{~cm}$; green $=4.5 \mu \mathrm{m}$; red $=8 \mu \mathrm{m}$ ) of the studied region. 


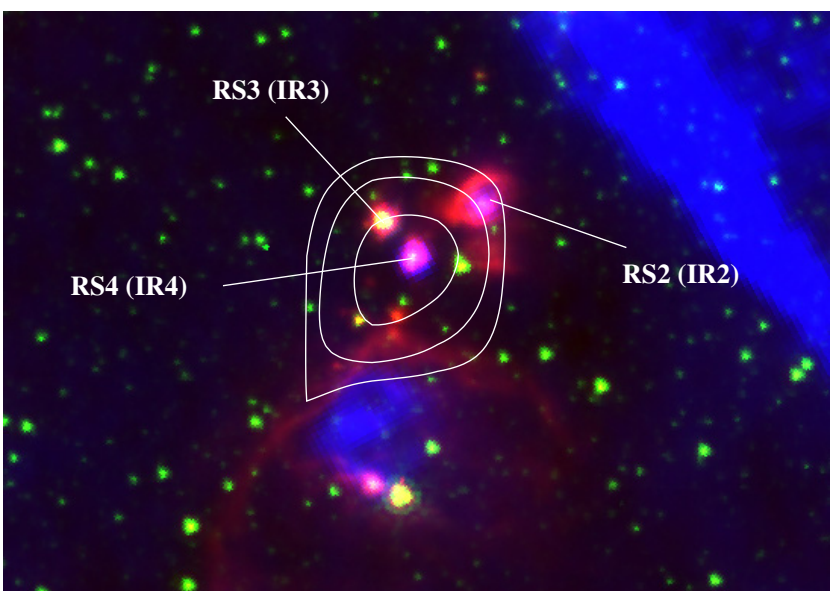

Fig. 11. Three-color image (blue $=$ radio continuum at $20 \mathrm{~cm}$; green $=4.5 \mu \mathrm{m}$; red $=8 \mu \mathrm{m}$ ). The radio sources RS2, RS3, and RS4 are indicated, and between brackets the nomenclature of the associated infrared sources is indicated: WISE J182411.60-122726.0 (IR2), J182413.49-122730.0 (IR3), and J182412.89-122742.2 (IR4). The contours represent the ${ }^{13} \mathrm{CO} \mathrm{J}=3-2$ emission integrated between 10 and $30 \mathrm{~km} \mathrm{~s}^{-1}$ with levels of 10,15 , and $30 \mathrm{~K} \mathrm{~km} \mathrm{~s}^{-1}$.

The contours represent the ${ }^{13} \mathrm{CO} J=3-2$ emission integrated from 10 to $30 \mathrm{~km} \mathrm{~s}^{-1}$ with levels of 15,20 , and $30 \mathrm{~K} \mathrm{~km} \mathrm{~s}^{-1}$. The radio sources, RS2, RS3, and RS4 (see Sect. 2) are seen in projection onto the molecular gas condensation. The brightest one in the radio band, RS4, positionally coincides with the peak of the ${ }^{13} \mathrm{CO} J=3-2$ emission, while RS2 is located towards the northwestern border of the clump. From the figure it can be noticed that each radio source has $8 \mu \mathrm{m}$ emission associated with an excellent spatial correlation, which confirms the thermal nature of the radio continuum emission. As noticed above, Giveon et al. (2005) identified RS4 as a young compact HII region. In this work we identify two new likely young HII regions, RS2 (G018.765+0.262) and RS3 (G018.762+0.270). While in the case of RS3 the radio continuum and the $8 \mu \mathrm{m}$ emission have the same compact structure, in RS2 the $8 \mu \mathrm{m}$ emission exhibits a shell-like structure that encircles the radio continuum emission.

From the WISE All-Sky Source Catalog (Wright et al. 2010), we searched for infrared sources related to radio ones. We identified the sources WISE J182411.60-122726.0 (IR2), J182413.49122730.0 (IR3), and J182412.89-122742.2 (IR4), which are related to the radio sources RS2, RS3, and RS4, respectively.

We performed a fitting of the spectral energy distribution (SED) of IR2, IR3, and IR4, using the tool developed by Robitaille et al. $(2007)^{3}$ and adopting an interstellar extinction in the line of sight, $A_{v}$, between 14 and 50 mag. The lower limit of $A_{v}$ was chosen by considering the typical value of one magnitude of absorption per kpc. The upper limit corresponds to that of $A_{v}$ derived from the molecular material, which was estimated by using the relation $A_{v}=\frac{N\left(\mathrm{H}_{2}\right)}{0.94 \times 10^{21}}$ mag (Frerking et al. 1982), with the $N\left(\mathrm{H}_{2}\right)$ derived in Sect. 5. In Fig. 12 we show the SEDs for the three sources with the best-fitting model for each source, and the subsequent good fitting models with $\chi^{2}-\chi_{\text {best }}^{2}<3$ (where $\chi_{\text {best }}^{2}$ is the $\chi^{2}$ per data point of the best-fitting model for each source). To construct the SED we consider the fluxes at the WISE 3.4, 4.6, 12, and $22 \mu \mathrm{m}$ bands. Table 2 presents the main physical parameters of each source as obtained from the bestfitting model and the range from the subsequent models: star

\footnotetext{
3 http://caravan.astro.wisc.edu/protostars/
}
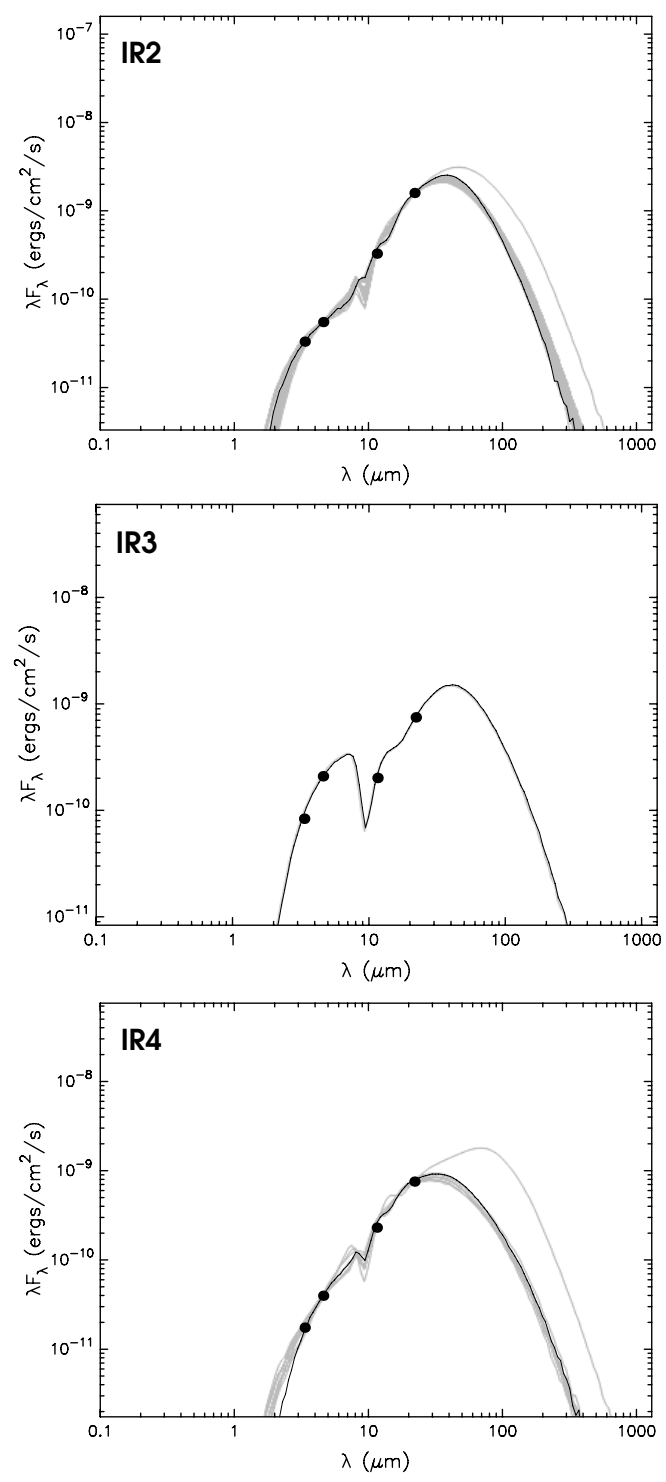

Fig. 12. SED models for IR2, IR3, and IR4. The circles indicate the measured fluxes. Black and gray solid curves represent the best-fit model and the subsequent good fittings models (with $\chi^{2}-\chi_{\text {best }}^{2}<3$ ), respectively.

mass (Col. 2), star age (Col. 3), envelope accretion rate (Col. 4), bolometric luminosity (Col. 5), $\chi^{2}$ per data point of the bestfitting model (Col. 6), and the number of subsequent good fitting models (Col. 7).

From the SED we conclude that the three sources are massive protostars. The derived ages are consistent with presence of ionized gas as detected in the radio continuum emission at $20 \mathrm{~cm}$. It is well known that the formation of an ultracompact HII region around a massive protostar requires timescales of about $10^{5} \mathrm{yr}$ (Sridharan et al. 2002). The analysis of the SED also shows that IR2 seems to be the most evolved among the three sources. While IR3 and IR4 are protostars that are still accreting material at high rates $\left(\dot{M}_{\text {env }}>5 \times 10^{-5} M_{\odot} \mathrm{yr}^{-1}\right)$, IR2 probably finished its accretion stage $\left(\dot{M}_{\text {env }} \sim 0\right)$. Moreover, from Fig. 11 it can be seen a shell-like morphology at $8 \mu \mathrm{m}$ towards this source, which shows the border of an incipient photodissociation region. By comparing the age of these young stellar objects (YSO; see Table 2) with that of the SNR (about $10^{5} \mathrm{yr}$ ), we conclude that the formation of the YSOs should be approximately coeval with the SN explosion, discarding the possibility that the SNR had 
Table 2. Main physical parameters of IR2, IR3, and IR4 derived from the best fitting models for the SED of each source.

\begin{tabular}{|c|c|c|c|c|c|c|c|c|c|c|}
\hline \multirow[t]{2}{*}{ Source } & \multicolumn{2}{|c|}{$\begin{array}{c}M_{\star} \\
{\left[M_{\odot}\right]}\end{array}$} & \multicolumn{2}{|c|}{$\begin{array}{c}\text { Age } \\
{\left[\times 10^{5} \mathrm{yr}\right]}\end{array}$} & \multicolumn{2}{|c|}{$\begin{array}{c}\dot{M}_{\mathrm{env}} \\
{\left[\times 10^{-5} M_{\odot} \mathrm{yr}^{-1}\right]}\end{array}$} & \multicolumn{2}{|c|}{$\begin{array}{c}L \\
{\left[\times 10^{4} L_{\odot}\right]}\end{array}$} & \multirow[t]{2}{*}{$\chi_{\text {best }}^{2}$} & \multirow[t]{2}{*}{$\begin{array}{c}\# \text { models } \\
\chi^{2}-\chi_{\text {best }}^{2} \leq 3\end{array}$} \\
\hline & Best & Range & Best & Range & Best & Range & Best & Range & & \\
\hline IR2 & 19 & $18-21$ & 7 & $1-10$ & 0 & $0-0.1$ & 4 & $2-5$ & 0.1 & 42 \\
\hline IR3 & 17 & - & 1 & - & 9.3 & - & 3 & - & 5.2 & 1 \\
\hline IR4 & 13 & $10-16$ & 2 & $0.5-4$ & 5.6 & $1-8$ & 1 & $1-2$ & 0.4 & 11 \\
\hline
\end{tabular}

triggered the star formation in the region. It it important to note that, if the SED is performed using the near distances of $1-3 \mathrm{kpc}$, we find that the sources should be very young (ages of about $\left.10^{4} \mathrm{yrs}\right)$ with low masses $\left(4-6 M_{\odot}\right)$; stellar sources with these parameters are unable to ionize the gas and generate an HII region complex.

\section{Concluding remarks}

In this work we have presented the results from molecular observations $\left({ }^{12} \mathrm{CO}\right.$ and ${ }^{13} \mathrm{CO} J=3-2$, and $\mathrm{HCO}^{+} J=4-3$ ) obtained with ASTE towards a molecular clump that belongs to an extended cloud supposedly interacting with the eastern flank of the SNR G18.8+0.3. Our observational study was complemented using IR, submillimeter, and radio continuum data from public databases.

The surveyed region contains four radio sources, two of them cataloged as HII regions: G18.751+0.254 (RS1 in this work) and a compact one (RS4). The rest of the sources, RS2 and RS3, were identified as new young HII regions. We found a molecular clump, seen mainly in the ${ }^{12} \mathrm{CO} J=3-2$ line, partially surrounding the HII region $\mathrm{G} 18.751+0.254(\mathrm{RS} 1)$ with its densest portion located to the north (seen at ${ }^{13} \mathrm{CO} J=3-2$ and $\mathrm{HCO}^{+}$ $J=4-3$ ). This densest portion coincides with the $1.1 \mathrm{~mm}$ continuum source BGPS 18.763+00.261, and the sources RS2, RS3, and RS4 are embedded in it.

The SNR G18.8+0.3, together with the radio sources RS1 and RS4, and the molecular gas have the same LSR velocity of about $20 \mathrm{~km} \mathrm{~s}^{-1}$. We concluded that all the analyzed objects are located at the same distance of about $14 \pm 1 \mathrm{kpc}$. An important result is that the new molecular observations reveal that the surveyed molecular clump is not in physical contact with the SNR, suggesting that either the SNR shock front has not yet reached the clump or they are located at different distances.

We identified the IR counterparts of the radio sources RS2, RS3, and RS4, those sources embedded in the densest portion of the molecular clump. From an SED fitting we conclude that they are YSOs with ages of about $10^{5} \mathrm{yr}$, which is consistent with the presence of ionized gas as seen in the radio continuum emission at $20 \mathrm{~cm}$. Their formation should be approximately coeval with the $\mathrm{SN}$ explosion, thereby discarding triggered star formation by the action of the SNR.
Acknowledgements. The ASTE project is led by Nobeyama Radio Observatory (NRO), a branch of National Astronomical Observatory of Japan (NAOJ), in collaboration with University of Chile, and Japanese institutes including University of Tokyo, Nagoya University, Osaka Prefecture University, Ibaraki University, Hokkaido University, and the Joetsu University of Education. S.P., M.O., G.D. and E.G. are members of the Carrera del investigador científico of CONICET, Argentina. A.P. is a doctoral fellow of CONICET. This work was partially supported by Argentina grants awarded by UBA, CONICET, and ANPCYT. M.R. wishes to acknowledge support from FONDECYT (CHILE) grant No. 108033. She is supported by the Chilean Center for Astrophysics FONDAP No. 15010003. S.P. and M.O. are grateful to the ASTE staff for the support received during the observations.

\section{References}

Anderson, L. D., Bania, T. M., Balser, D. S., \& Rood, R. T. 2011, ApJS, 194, 32 Arnal, E. M., Dubner, G., \& Goss, W. M. 1993, Rev. Mex. Astron. Astrofis., 26, 93

Bally, J., Aguirre, J., Battersby, C., et al. 2010, ApJ, 721, 137

Churchwell, E., Povich, M. S., Allen, D., et al. 2006, ApJ, 649, 759

Churchwell, E., Watson, D. F., Povich, M. S., et al. 2007, ApJ, 670, 428

Cortes, P. C. 2011, ApJ, 743, 194

Cortes, P. C., Parra, R., Cortes, J. R., \& Hardy, E. 2010, A\&A, 519, A35

Dubner, G., Giacani, E., Reynoso, E., et al. 1999, AJ, 118, 930

Dubner, G., Giacani, E., Reynoso, E., \& Parón, S. 2004, A\&A, 426, 201

Dubner, G. M., Giacani, E. B., Goss, W. M., Moffett, D. A., \& Holdaway, M. 1996, AJ, 111, 1304

Dunham, M. K., Rosolowsky, E., Evans, II, N. J., Cyganowski, C., \& Urquhart, J. S. 2011, ApJ, 741, 110

Ezawa, H., Kawabe, R., Kohno, K., \& Yamamoto, S. 2004, in SPIE Conf. 5489, ed. J. M. Oschmann, Jr., 763

Frerking, M. A., Langer, W. D., \& Wilson, R. W. 1982, ApJ, 262, 590

Giveon, U., Becker, R. H., Helfand, D. J., \& White, R. L. 2005, AJ, 129, 348

Helfand, D. J., Becker, R. H., White, R. L., Fallon, A., \& Tuttle, S. 2006, AJ, 131,2525

Milam, S. N., Savage, C., Brewster, M. A., Ziurys, L. M., \& Wyckoff, S. 2005, ApJ, 634, 1126

Robitaille, T. P., Whitney, B. A., Indebetouw, R., \& Wood, K. 2007, ApJS, 169, 328

Rosolowsky, E., Dunham, M. K., Ginsburg, A., et al. 2010, ApJS, 188, 123

Savage, C., Apponi, A. J., Ziurys, L. M., \& Wyckoff, S. 2002, ApJ, 578, 211

Schuller, F., Menten, K. M., Contreras, Y., et al. 2009, A\&A, 504, 415

Scoville, N. Z., Sargent, A. I., Sanders, D. B., et al. 1986, ApJ, 303, 416

Simon, R., Jackson, J. M., Clemens, D. P., Bania, T. M., \& Heyer, M. H. 2001, ApJ, 551, 747

Sridharan, T. K., Beuther, H., Schilke, P., Menten, K. M., \& Wyrowski, F. 2002, ApJ, 566, 931

Tian, W. W., Leahy, D. A., \& Wang, Q. D. 2007, A\&A, 474, 541

Wood, D. O. S., \& Churchwell, E. 1989, ApJ, 340, 265

Wright, E. L., Eisenhardt, P. R. M., Mainzer, A. K., et al. 2010, AJ, 140, 1868 